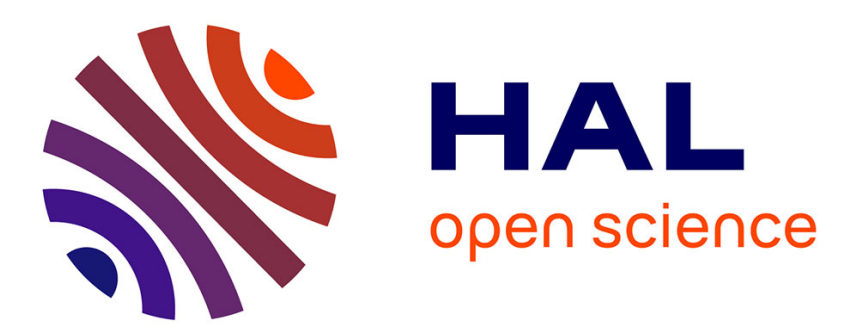

\title{
Numerical and analytical estimation of the ageing linear viscoelastic behavior of a two-phase composite with expansive inclusions
}

\author{
Tulio Honorio, B. Bary
}

\section{- To cite this version:}

Tulio Honorio, B. Bary. Numerical and analytical estimation of the ageing linear viscoelastic behavior of a two-phase composite with expansive inclusions. FraMCoS-X - 10th International Conference on Fracture Mechanics of Concrete and Concrete Structures, Jun 2019, Bayonne, France. 10.21012/FC10.235550 . hal-02163047

\section{HAL Id: hal-02163047 \\ https://hal.science/hal-02163047}

Submitted on 23 Jun 2019

HAL is a multi-disciplinary open access archive for the deposit and dissemination of scientific research documents, whether they are published or not. The documents may come from teaching and research institutions in France or abroad, or from public or private research centers.
L'archive ouverte pluridisciplinaire HAL, est destinée au dépôt et à la diffusion de documents scientifiques de niveau recherche, publiés ou non, émanant des établissements d'enseignement et de recherche français ou étrangers, des laboratoires publics ou privés. 


\title{
NUMERICAL AND ANALYTICAL ESTIMATION OF THE AGEING LINEAR VISCOELASTIC BEHAVIOR OF A TWO-PHASE COMPOSITE WITH EXPANSIVE INCLUSIONS
}

\author{
T. HONORIO ${ }^{\dagger}$ AND B. BARY * \\ $\dagger$ LMT, ENS Cachan, CNRS, Université Paris-Saclay, F-94235 Cachan, France \\ email : tulio.honorio-de-faria@ens-paris-saclay.fr \\ * Den-Service d'Etude du Comportement des Radionucléides (SECR), CEA, Université Paris-Saclay, F- \\ 91191, Gif-sur-Yvette, France \\ e-mail: benoit.bary@cea.fr
}

Key words: Levin formula, Homogenization, Creep, Composites, Durability

\begin{abstract}
We investigate the behavior of a two-phase composite consisting of an inclusive elastic phase and an ageing linear viscoelastic matrix (ALVE) experiencing an expansion of the inclusions or equivalently a differential dilation of both phases. We propose, based on an analogy with thermoviscoelasticity, to estimate the macroscopic mechanical properties of the composite and to determine its overall free strains and macroscopic coefficient of expansion. Numerically, 3D cubic specimens are generated with spherical or polyhedral inclusion shapes. Finite element simulations are then performed, allowing to calculate the macroscopic strains generated by the expansions, as well as the moduli at different time of loading. The effect of the inclusions shape on both local and macroscopic behavior are also studied. Analytically, a methodology suitable for estimating the overall behavior of composite materials in an ALVE context is applied, and extended to the case of ageing linear thermoviscoelasticity. Specifically, the macroscopic effects of the expansion of inclusions assumed as spherical are determined via the dilation coefficient, and obtained by a semi-explicit homogenization procedure. Finally, numerical and analytical results are compared in terms of macroscopic response and mechanical properties at different ages, and for various ageing and expansion functions.
\end{abstract}

\section{INTRODUCTION}

Understanding creep in cement-based materials is crucial to predict the performance of concrete infrastructures. Due to hydration processes at early-age or degradation processes at late ages, both changing the microstructure of the material, concrete exhibits an ageing behavior. Numerical and analytical homogenization approaches have been developed to upscale the effective properties of composites in an ageing linear viscoelasticity (ALVE) [1]-[3].
Creep in concrete is known to be sensible to temperature and relative humidity. Therefore, formulations accounting for the transformation field induced by temperature and relative humidity changes are needed to upscale the viscoelastic behavior of concrete in changing environments. Also, transformation fields are also encountered in the case of chemical attacks associated with a secondary phase precipitation exerting a pressure on pore walls, or due to an external source of neutron radiation provoking the expansion of aggregates and a degradation 
of the matrix mechanical properties. However, accounting for transformation fields analytically in ALVE is challenging since Hill lemma needs to be proved valid in this context [4]. Another strategy, based on an early proposition of Levin [5], [6] regarding exact solution for composites with two isotropic phases, has not yet been explored.

In this study, we first extend Levin formula to the case of ALVE. Then we estimate, using both analytical and numerical homogenization approaches, the effective behaviour of a twophase composite consisting of elastic inclusions and an ageing linear viscoelastic matrix, experiencing a progressive expansion of the inclusions or a differential matrix/inclusion dilation. All phases are considered isotropic; the matrix phase is ruled by a linear viscoelastic behaviour whose moduli are affected by an ageing function. Potential damage of the matrix is not considered in this study. Growth is described by an equivalent thermal expansion. Both aggregate expansion and ageing of the matrix phase are assumed to be time-dependent functions. Numerically, 3D cubic specimens are generated with spherical or polyhedral inclusion shapes [7], [8]. Simulations are then performed with the finite element code Cast3M developed at CEA (www-cast3m.cea.fr), allowing to calculate the macroscopic strains generated by the expansions, as well as the moduli at different time of loading. The effect of the inclusions shape on both local and macroscopic behaviour are also studied. Analytically, a methodology suitable for estimating the overall behaviour of composite materials in an ALVE context is applied [1], and extended to the case of ageing linear thermoviscoelasticity. Specifically, the macroscopic effects of the expansion of inclusions assumed as spherical are determined via an interaction coefficient analogous to the dilation coefficient, and obtained by a semiexplicit homogenization procedure. Finally, numerical and analytical results are compared in terms of macroscopic response and mechanical properties at different ages, and for various ageing and expansion functions.

\section{ANALYTICAL SOLUTION FOR A COMPOSITE WITH TWO ISOTROPIC PHASES}

In this section we propose an analytical solution for the problem of the thermal expansion in a ALVE composite of two isotropic phases based on the corresponding derivation in elasticity [5]. Let the thermal problem in ALVE be written in the form:

$$
\begin{aligned}
& \boldsymbol{\varepsilon}(\mathbf{x}, t)= \\
& \mathbf{S}(\mathbf{x}, t, .) \stackrel{\circ}{:} \boldsymbol{\sigma}(\mathbf{x}, .)+\boldsymbol{\alpha}(\mathbf{x}, t, .) \circ \theta(.)
\end{aligned}
$$

where the time dependent strain field $\boldsymbol{\varepsilon}$ can be written as a function of the time dependent stress field $\boldsymbol{\sigma}$, compliance tensor $\mathbf{S}$, thermal expansion tensor $\boldsymbol{\alpha}$ and the variation in the temperature $\theta=T-T_{0}$; and $\stackrel{\circ}{\boldsymbol{m}}$ designates the integral operator $(f \stackrel{\circ}{\sim} g)\left(t, t_{0}\right) \equiv$ $\int_{t^{\prime}=-\infty}^{t} f\left(t, t^{\prime}\right)=d_{t^{\prime}} g\left(t^{\prime}, t_{0}\right)$, where - is a product to be defined. Note that the Heaviside function $H$ acts as the multiplicative identity with respect to the integral operator "。" (i.e. $f \circ f^{\stackrel{\circ}{-1}}=H$ ). This formalism was deployed to derive homogenization schemes in ALVE [1], [2]. The integral operator can be evaluated using numerical integration [1].

The average mechanical fields are related by the following expression written in terms of effective compliance $\mathbf{S}^{*}$ and thermal expansion tensor $\boldsymbol{\alpha}^{*}$ :

$$
\begin{aligned}
& \langle\boldsymbol{\varepsilon}(t)\rangle \\
& =\mathbf{S}^{*}(t, .) \stackrel{\circ}{:}\langle\boldsymbol{\sigma}(.)\rangle+\boldsymbol{\alpha}^{*}(t, .) \circ \theta(.)
\end{aligned}
$$

We are interested in a solution where the stress field is a uniform hydrostatic pressure $p$ [5]:

$$
\boldsymbol{\sigma}(\mathbf{x}, t)=\langle\boldsymbol{\sigma}(t)\rangle=-p(t) \mathbf{I}
$$

for all $\mathbf{x}$, where is the identity $3 \times 3$ matrix.

In a medium constituted of two phases (denoted by subscripts $i=1$ and 2):

$$
\begin{aligned}
& \boldsymbol{\varepsilon}_{i}(t)= \\
& \mathbf{S}_{i}(t, .) \stackrel{\circ}{:}(-p(.) \mathbf{I})+\boldsymbol{\alpha}_{i}(t, .) \circ \theta(.)
\end{aligned}
$$

Thus, the strain field is completely uniform (i.e. $\boldsymbol{\varepsilon}_{\mathbf{1}}(t)=\boldsymbol{\varepsilon}_{\mathbf{2}}(t)=\langle\boldsymbol{\varepsilon}(t)\rangle$ ) if 


$$
\begin{aligned}
& p(t) \circ \theta(.)^{\stackrel{\circ}{-1}}= \\
& \left(\mathbf{S}_{\mathbf{1}}-\mathbf{S}_{\mathbf{2}}\right)^{\stackrel{\sim}{-1}}(t, .) \stackrel{\circ}{\stackrel{m}{:}} \mathbf{I} \stackrel{\circ}{:}^{:}\left(\boldsymbol{\alpha}_{2}-\boldsymbol{\alpha}_{\mathbf{1}}\right)(t, .)
\end{aligned}
$$

Substituting (4) and (5) in (2), we obtain the Levin expression of the effective thermal expansion extended to the case of a composite with two isotropic ALVE phases:

$$
\begin{aligned}
& \boldsymbol{\alpha}^{*}(t)=\boldsymbol{\alpha}_{\mathbf{1}}(t)+ \\
& \left(\mathbf{S}^{*}-\mathbf{S}_{\mathbf{1}}\right)(t, .) \stackrel{\circ}{\stackrel{\sim}{:}}\left(p(t) \circ \theta(.)^{\stackrel{\circ}{-1}}\right) \mathbf{I} \\
& =\boldsymbol{\alpha}_{2}(t) \\
& +\left(\mathbf{S}^{*}-\mathbf{S}_{2}\right)(t, .) \stackrel{\circ}{\stackrel{\circ}{:}}\left(p(t) \circ \theta(.)^{\stackrel{\circ}{-1}}\right) \mathbf{I}
\end{aligned}
$$

As the original Levin formula, this expression is microstructure independent. Once the effective elastic properties of a micro- and macro-isotropic composite is known, this formula can be used to exactly determine the CTE of the composite. Besides thermomechanical problems, this expression can also be used to upscale the coupling parameter related to other transformations fields that can be described by the linearized form in Eq. (1), such as the ones found in poromechanics, or due to expansive phases.

In following, Mori-Tanaka estimations, which are adapted to a matrix-inclusion morphology, for isotropic spherical inclusions in ALVE [1] will be deployed.

\section{NUMERICAL SIMULATIONS}

We briefly describe in this section the $3 \mathrm{D}$ numerical simulations that have been performed to compare with the analytical estimations. More details can be found in e.g. [2], [7], [8]. The microstructures have been generated with the Combs python tool using the CAD code Salome (www.salomeplatform.org). It mainly consists in randomly placing inclusions of specified shape and size in a box whose dimensions are also fixed by the user. Non-overlapping of particles is enforced by imposing a minimal distance between them during the placement, while they are free to cross the box faces. Here, two types of microstructures were generated with identical particle volume fraction of $40 \%$, one with aggregates obtained by a Voronoi space decomposition, the other with spherical inclusions. The objective is to evaluate the effects of rounded versus angular shapes on the results. The Figure 1 presents the geometry of the generated mesostructures. The meshes are composed of $2.42 \times 10^{6}$ and $3.13 \times 10^{6}$ tetrahedral elements for the samples with Voronoi and spherical inclusions, respectively. The mechanical loading is applied via classical homogeneous stresses boundary conditions, and the thermal loading consists in homogeneous temperature imposed (in general instantaneously) throughout the samples. The simulations allow obtaining macroscopic as well as local (i.e. averaged per phase, per inclusion or at prescribed positions) information in terms of stresses and strains.
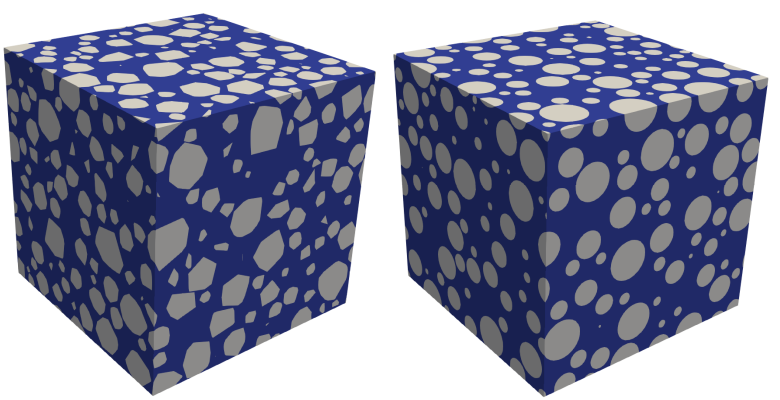

Figure 1: 3D microstructures analysed, with Voronoi (left) and spherical (right) particles.

\section{RESULTS}

The behavior of the matrix is represented by a generalized Maxwell model:

$$
X\left(t-t^{\prime}\right)=x_{0}+\sum_{i} x_{i} \exp \left[-\left(t-t^{\prime}\right) / \chi_{i}\right]
$$

where $X$ is the shear or bulk relaxation functions, $\mu$ and $k$, respectively; $x_{i}$ are the parameters of generalized Maxwell model associated with each relaxation function and $\chi_{i}$ is the characteristic time of each chain. We use the parameters obtained for ageing mortars [8], which are gathered in Table 1. The inclusions are elastic with a Young modulus of $70 \mathrm{GPa}$ and Poisson ratio of 0.3 . The volume fraction of inclusions is $40 \%$. 

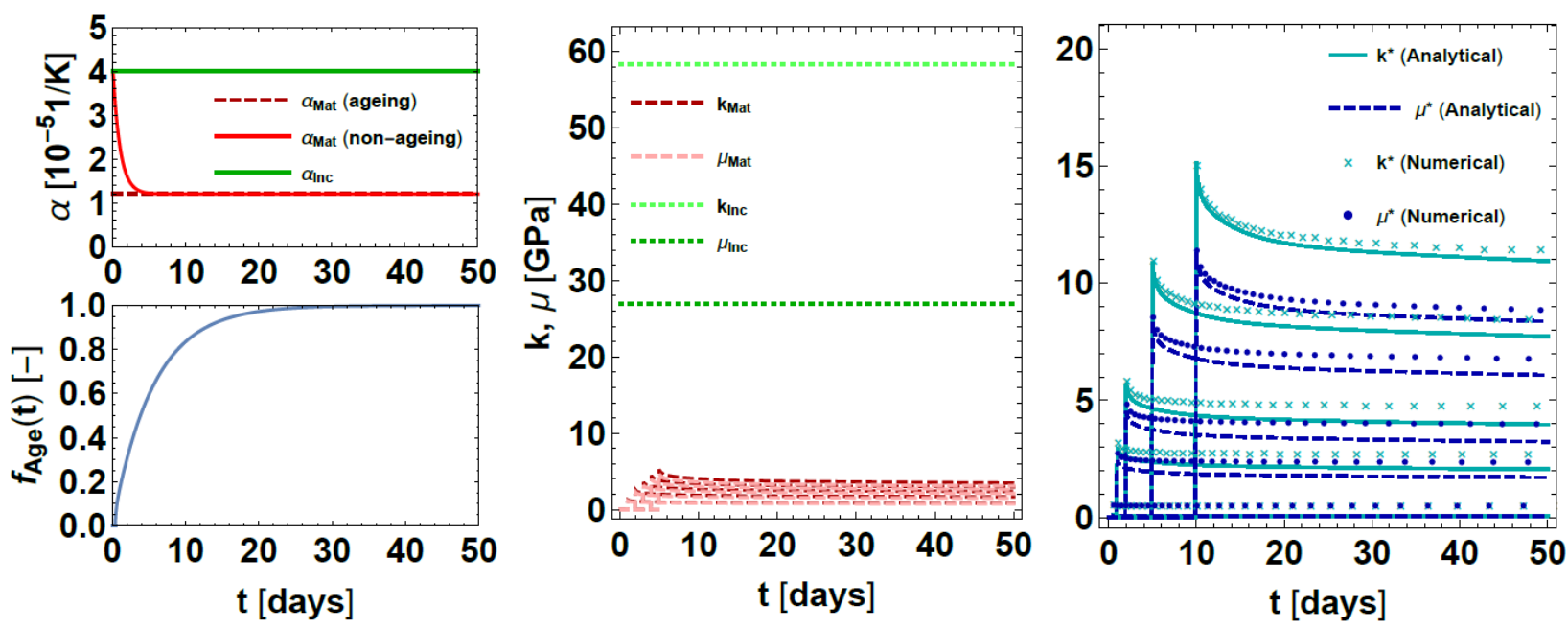

Figure 2: At the left, coefficients of thermal expansion of the constituents (an ageing and a constant $\alpha_{M a t}$ are considered) and ageing function $f_{A g e}$ associated with the shear or bulk relaxation functions. At the center, shear and bulk moduli of the inclusion and shear and bulk relaxation functions of the matrix (these functions are ageing following $f_{\text {Age }}$ ). At the right, effective shear or bulk relaxation functions: estimations obtained analytical (full lines) and numerical (symbols, only the numerical sample with spherical inclusions is considered) homogenization.

The coefficient of thermal expansion of the inclusions is $\alpha_{\text {Inc }}=4.0 \times 10^{-5} 1 / \mathrm{K}$. For the matrix we consider (i) a constant $\left(\alpha_{\text {Mat }}=\right.$ $\left.1.2 \times 10^{-5} 1 / \mathrm{K}\right)$ and (ii) an ageing $\left(\alpha_{\text {Mat }}(t)=\right.$ $\left.\left(2.8 e^{-t}+1.2\right) \times 10^{-5} 1 / \mathrm{K}\right) \quad$ coefficient of thermal expansion, as depicted in Figure 2 (left).

Table 1: Properties of the matrix based on Maxwell generalized behavior

\begin{tabular}{cccc}
\hline & $\begin{array}{c}k \\
{[\mathrm{GPa}]}\end{array}$ & $\begin{array}{c}\mu \\
{[\mathrm{GPa}]}\end{array}$ & $\begin{array}{c}\chi \\
{[\mathrm{days}]}\end{array}$ \\
\hline$x_{0}$ & 3.9211 & 3.3754 & - \\
\hline$x_{1}$ & 0.5785 & 0.4772 & 0.966 \\
\hline$x_{2}$ & 1.0525 & 0.9658 & 10 \\
\hline$x_{3}$ & 1.5282 & 1.3853 & 100 \\
\hline$x_{4}$ & 0.6480 & 0.5783 & 1000 \\
\hline$x_{5}$ & 2.9801 & 2.7789 & 10000 \\
\hline
\end{tabular}

The effective bulk and shear relaxation functions obtained from both numerical and analytical homogenization are shown in Figure 2 (right) for different ages of loading. Both estimations yield consistent results; we note however that the numerical curves are always (slightly) higher than the analytical ones.

Figure 3 shows the effective coefficients of thermal expansion obtained from both numerical and analytical homogenization for a constant and an ageing thermal expansion of the matrix (following Fig. 2 (left)). In the former case, the effective coefficient is approximately constant in spite of the ageing aspect of the bulk and shear relaxation functions. By contrast, it is ageing in the case in which the matrix itself has an ageing coefficient of thermal expansion. In both cases, the effective coefficient of thermal expansion is also viscoelastic, with a characteristic time for the relaxation of the response, as can be seen in the Figure 4 presenting the analytical results, on the order of few days. Note that the numerical estimations of the effective coefficient are systematically higher than the analytical ones. This may be explained by the effective moduli that are greater in the simulations than in the analytical case, see Figure 2 (right). Another remark is that the effective coefficients of thermal expansion computed with spherical and Voronoi aggregates are close, meaning that the effect of the particle shape as considered here is weak.

Figure 4 also shows that the effective coefficient in the case of a matrix with ageing thermal expansion coefficient retrieves the one in the case of a matrix with non-ageing thermal 
after few days, that is for loading times that exceed the characteristic time of $\alpha_{M a t}(t)$ (i.e on the order of 1 day).
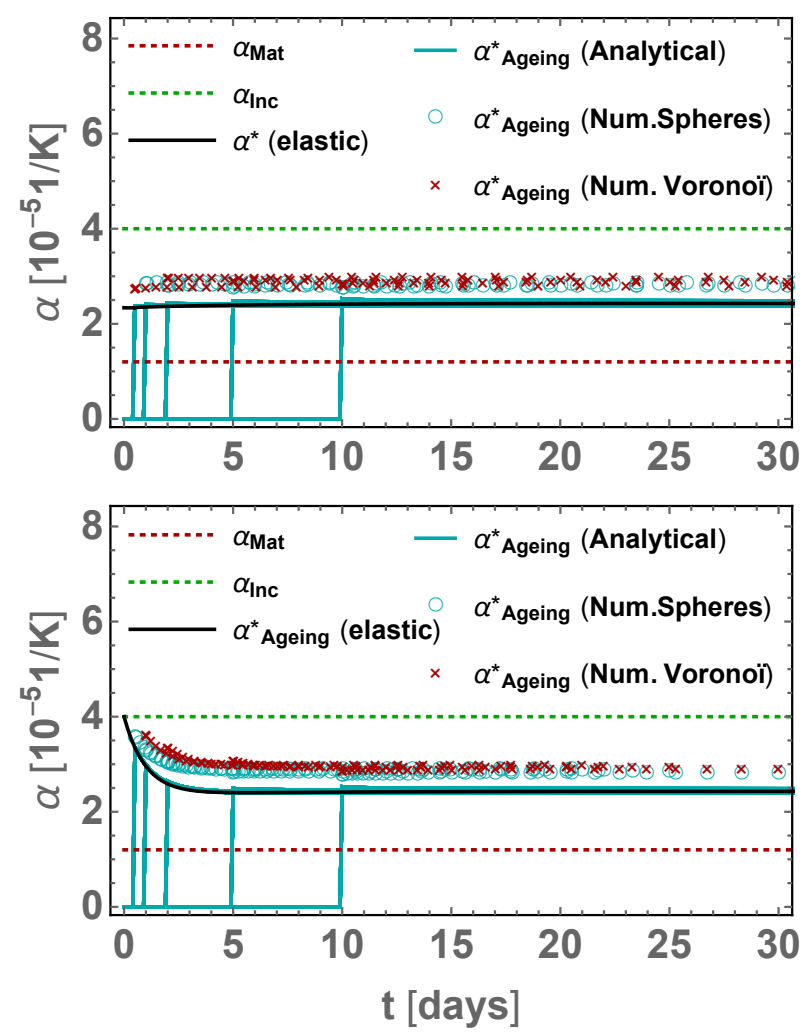

Figure 3: Effective coefficients of thermal expansion considering a constant (top) and an ageing (bottom) thermal expansion coefficient for the matrix. Both analytical and numerical estimations are shown (for the latter, we compare the estimations regarding spherical and Voronoï aggregates).

Figure 5 presents the FE average stresses developed in both phases in the case of the microstructure with spherical inclusions and ageing thermal expansion coefficient, for different ages of thermal loading consisting in an (instantaneous) increase of temperature of $100^{\circ} \mathrm{C}$. We observe as expected that the inclusion phase is in compression while the matrix one is in tension. For the latter, the stress level is all the higher than the age of loading is great; for ages superior to 1 day, it is believed that microcracking may appear due to the stress intensity. It is worth noting that in the case of late loading ages the stress evolutions are due essentially to the ageing viscoelasticity of the matrix behavior since the thermal expansion coefficient is almost constant.

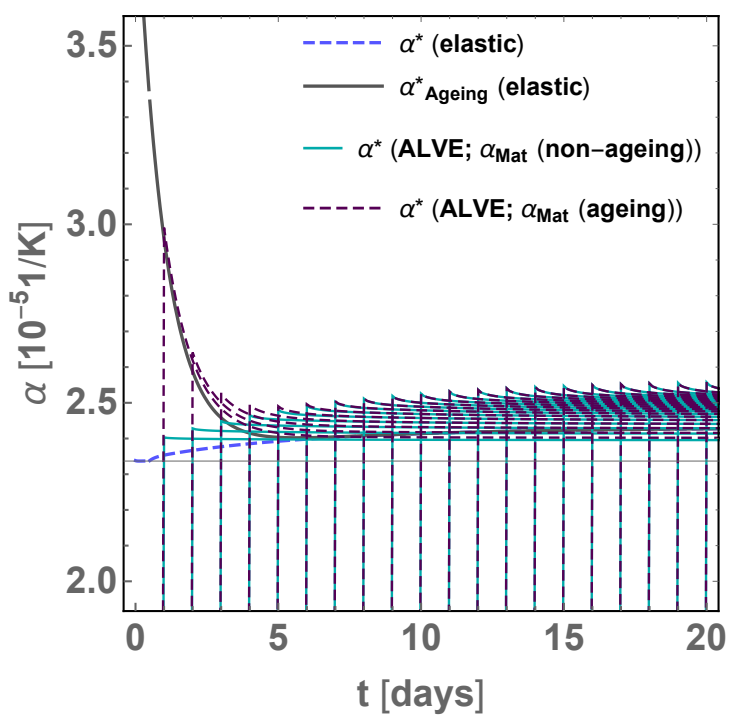

Figure 4: Effective coefficients of thermal expansion estimated by analytical homogenization: the mismatch between the characteristic times associated with $\alpha_{\text {Mat }}(t)$ and the shear and bulk relaxation functions leads to a "master curve" joining the peaks of the viscoelastic response that is non-monotonous.

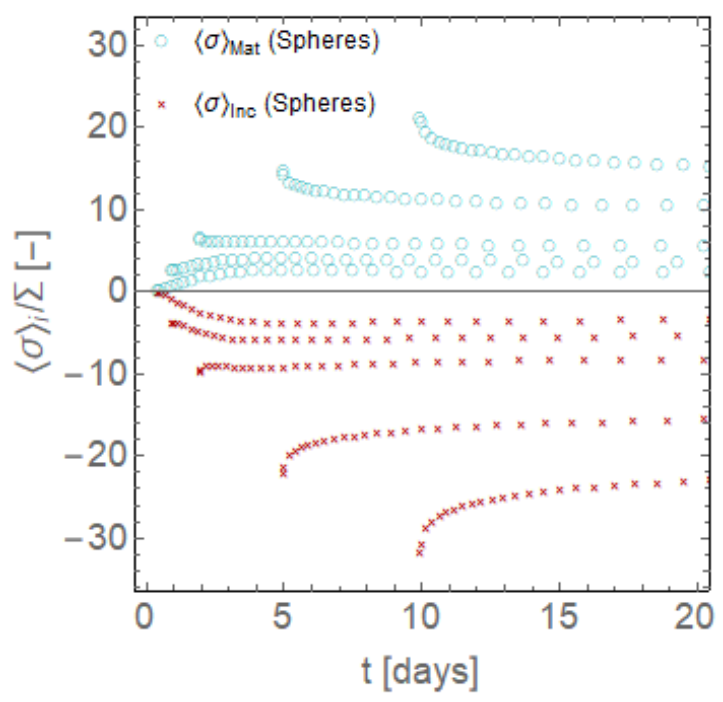

Figure 5: Computed average stresses in both phases in the spherical case and ageing thermal expansion coefficient, at different ages of thermal loading (increase of temperature of $100^{\circ} \mathrm{C}$, free macroscopic strains).

Finally, Figure 6 presents the estimated evolutions of the macroscopic expansion coefficient $\left(\alpha^{\mathrm{Rad}}\right)^{*}$ when the aggregates are subjected to an expansion of $\left(\alpha^{\mathrm{Rad}}\right)_{\text {Inc }}$. Here this expansion is supposed to be (linearly) dependent on time, as can be encountered e.g. in the case of a progressive neutron irradiation. 
The matrix phase behavior is the same as in the previous applications, i.e. ALVE. We observe a slight influence of the matrix viscoelasticity on $\left(\alpha^{\mathrm{Rad}}\right)^{*}$ as a function of the loading age, before it recovers a linear increase following the aggregate expansion. In this case, the effects of the irradiation on the matrix behavior and the microcrack development should also be considered in the future to be more realistic.

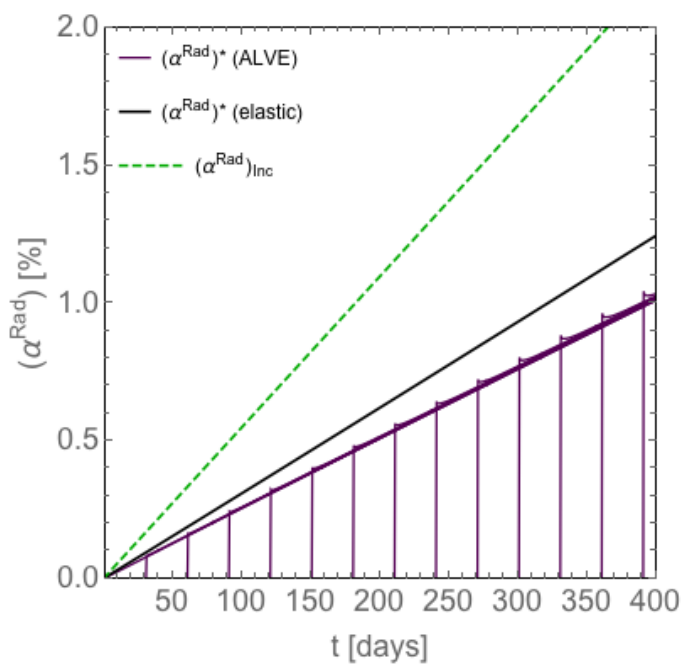

Figure 6: Estimation of the macroscopic expansion coefficient obtained for an aggregate expansion $\left(\alpha^{\mathrm{Rad}}\right)_{\text {Inc }}$ evolving linearly with time.

\section{CONCLUSIONS, PERSPECTIVES}

We have presented in this paper preliminary results obtained (semi-)analytically and numerically concerning the response of a twophase material subjected to the expansion of its constituents in the context of ALVE behavior. The expansion may be due e.g. to thermal loading or to the volume expansion of aggregates caused by irradiation or chemical alteration (precipitation of secondary phases). The analytical estimations are based on an extension of the Levin formula to the ALVE case, while the simulations are performed on 3D microstructures with the FE code Cast3M. Overall, the analytical estimations are fully consistent with the 3D simulations. Applications are provided assuming elastic aggregates and ALVE matrix. While the ageing behavior of the mechanical properties of the matrix influences the effective moduli, it is found that it has almost no influence on the effective expansion coefficient. By contrast, considering an ageing expansion coefficient for the matrix implies an ageing effective expansion coefficient. Finally, a first analytical application of a material submitted to a timedependent aggregate expansion is provided; the results show a slight influence of the viscoelasticity of the matrix. Further analyses and comparison with simulations remain to be done.

As perspectives, a throughout comparison of local information concerning average and fluctuations of mechanical fields per phase can be envisioned. Microcracking and the corresponding influence on the mechanical properties would also deserve to be considered, given the high level of stresses/strains that can develop in particular when aggregates expand.

\section{REFERENCES}

[1] J. Sanahuja, "Effective behaviour of ageing linear viscoelastic composites: Homogenization approach," Int. J. Solids Struct., vol. 50, no. 19, pp. 2846-2856, Sep. 2013.

[2] T. Honorio, B. Bary, J. Sanahuja, and F. Benboudjema, "Effective properties of ncoated composite spheres assemblage in an ageing linear viscoelastic framework," Int. J. Solids Struct., vol. 124, pp. 1-13, Oct. 2017.

[3] S. Scheiner and C. Hellmich, "Continuum microviscoelasticity model for aging basic creep of early-age concrete," J. Eng. Mech., vol. 135, no. 4, pp. 307-323, 2009.

[4] T. Honorio, "Micromechanics of transformation fields in ageing linear viscoelastic composites: effects of phase dissolution or precipitation," Mech. TimeDepend. Mater., pp. 1-16, Feb. 2017.

[5] G. W. Milton, The Theory of Composites. Cambridge: Cambridge University Press, 2002.

[6] V. M. Levin, “Thermal Expansion Coefficients of Heterogeneous Materials," Lzu Akad Nauk SSSR Mekh Tuerd Tela Vol 2, pp. 88-94, 1967.

[7] B. Bary, C. Bourcier, and T. Helfer, "Analytical and 3D numerical analysis of 
the thermoviscoelastic behavior of concrete-like materials including interfaces," Adv. Eng. Softw., vol. 112, pp. 16-30, Oct. 2017.

[8] T. Honorio, B. Bary, and F. Benboudjema, "Multiscale estimation of ageing viscoelastic properties of cement-based materials: A combined analytical and numerical approach to estimate the behaviour at early age," Cem. Concr. Res., vol. 85, pp. 137-155, Jul. 2016. 